Research Article

\section{Evaluation of the effect of coronary artery bypass grafting on the right ventricular function using speckle tracking echocardiography}

\author{
Mahmoud Shawky Abdelmoneum¹, Neama Ali Elmeligy², Elsayed \\ Abdelkhalek Eldarky ${ }^{3}$ and Mohamad Mahmoud Mohamad4* \\ ${ }^{1}$ Lecturer of Cardiology, Faculty of Medicine, Benha University, Egypt \\ ${ }^{2}$ Assistant Consultant of Cardiology, Faculty of Medicine, Benha University, Egypt \\ ${ }^{3}$ Professor of Cardiology, Faculty of Medicine, Benha University, Egypt \\ ${ }^{4}$ Cardiology Resident, National Heart Institute, Egypt
}

\section{Abstract}

Purpose: This was a prospective study conducted at Benha University hospital and National Heart Institute on one hundred patients undwent coronary artery bypass grafting (CABG) to evaluate the effect of $C A B G$ on the right ventricular (RV) function using speckle tracking echocardiography (STE).

Methods: All cases were subjected to detailed medical history, full physical examination, 12 leads electrocardiogram (ECG), routine laboratory tests including (complete blood picture, liver functions, renal functions and lipid profile) and echocardiography either conventional echocardiography or STE, all parameters obtained before and within 2 weeks after surgery.

Results: By conventional echocardiography there was statistically significant decrease in peak right ventricle systolic velociy (RVS') from $(12.76 \pm 1.72)$ to $(7.33 \pm 1.71)$ and tricuspid annular plane systolic excursion (TAPSE) from $(22.8 \pm 3.99)$ to $(13.77 \pm 4.63)$ among the studied patients after $C A B G$. While there was significant increase in right ventricle fractional area change (RVFAC) from $(44.69 \pm 3.25)$ to $(49.01 \pm 3.36)$. On the other hand, there was non-significant change in right ventricle end diastolic diameter (RVEDD) at mid-cavity from $(26.37 \pm 2.72)$ to $(26.53 \pm 2.72)$ and basal segment from $(36.05 \pm 2.98)$ to $(36.29 \pm 3.04)$, right ventricle stroke volume (RVSV) from $(65.44 \pm 7.02)$ to $(65.85 \pm 6.86)$ and right myocardial performance index (RMPI) from $(0.491 \pm 0.088)$ to $(0.498 \pm 0.086)$.

By STE There was statistically significant decrease in right ventricle global longitudinal strain (RVGLS) from (-20.63 to -14.1) after CABG. There was statistically significant decrease in right ventricle free wall longitudinal strain [apical decreased from (-23.73 to -13.7), mid-cavity decreased from (-25.76 to -11.53$)$, basal decreased from $(-20.39$ to -10.13$)$ and lateral wall declined from (-23.01 to -9.13$)]$. There was statistically significant decrease in interventricular septum longitudinal strain [apical decreased from (-19.77 to -10.06$)$, mid-cavity decreased from $(-17.81$ to -10.87$)$ and basal decreased from $(-15.89$ to -11.13$)]$. There was statistically significant increase in RV circumferential strain of lateral free wall from (-12.04 to -16.21), while there was non-significant change in RV circumferential strain of septum from $(-19.77 \pm 4.86)$ to $(-20.37 \pm$ 5.14).

Conclusion: Distorted RV geometry after CABG can lead to altered deformation parameters, in other words longitudinal functional parameters may underestimate RV function and the decrease in RVGLS was compensated by increase in circumferential strain of lateral free wall of $\mathrm{RV}$ without change in RVSV or RMPI. Therefor changes in deformation parameters should always be interpreted in relation to change in geometry.

\section{More Information}

*Address for Correspondence: Mohamad Mahmoud Mohamad, Cardiology Resident, National Heart Institute, Egypt, Tel: +2001000417606; Email: elfuouly@gmail.com

Submitted: 16 December 2019

Approved: 27 December 2019

Published: 30 December 2019

How to cite this article: Abdelmoneum MS Elmeligy NA, Eldarky EA, Mohamad MM. Evaluation of the effect of coronary artery bypass grafting on the right ventricular function using speckle tracking echocardiography. J Cardiol Cardiovasc Med. 2019; 4: 236-241.

DOI: dx.doi.org/10.29328/journal.jccm.1001075

Copyright: @ 2019 Abdelmoneum MS, et al. This is an open access article distributed under the Creative Commons Attribution License, which permits unrestricted use, distribution, and reproduction in any medium, provided the original work is properly cited.

Keywords: Coronary artery bypass grafting; Longitudinal strain; Right ventricle; Circumferential strain

W) Check for updates

OPEN ACCESS 


\section{Introduction}

Coronary artery disease (CAD) is the leading cause of death globally resulting in over 7 million deaths. This increased from 5.2 million deaths in 1990. It may affect individuals at any age but becomes dramatically more common at progressively older ages, with approximately a tripling with each decade of life. Males are affected more often than females [1].

Treatment of CAD with CABG has been associated with better outcome and longer survival than percutaneous coronary intervention (PCI) using either drug eluting or bare metal stents [2]. Extensive literature search reveals that on pump CABG causes more complete revascularization as compared to off pump CABG, on pump patients tend to have significantly higher frequencies of complete revascularization as compared to patients treated via Off pump method [3]. Incomplete revascularization has traditionally been associated with increased mortality, evidence for that was the study done by Jones \& Weintraub, [4], and showed that incomplete revascularization lead to decreased patient survival and associated with high incidence of recurrent angina. Therefor off pump patients have higher rates of repeat revascularizations as compared to on pump patients [3].

Speckle tracking echocardiography (STE) is a new diagnostic modality that enabling the assessment of deformation in the longitudinal, circumferential and radial planes and showes good correlation in different studies [5].

Analysis of myocardial strain by tissue Doppler is restricted along the axis parallel to the ultrasound beam and is thus limited by this angle dependence. By analyzing speckle motion, (STE) offers the opportunity to assess myocardial tissue velocity, strain and strain rate independently of cardiac translation and beam angle. STE is simple to perform, it requires only one cardiac cycle, The software only requires harmonic and high frame rate imaging [6].

Quantative evaluation of RV function and changes in its cavity area from end diastole to end systole can be evaluated echocardiographically [7].

\section{Aim of the work}

The aim of this study was to evaluate the effect of CABG on the RV function using speckle tracking echocardiography.

\section{Subjects and Methods}

\section{Study design and population}

This was a prospective study conducted at Benha University hospital and National Heart Institute from 6/2/2019 to $14 / 11 / 2019$ on one hundred patients underwent CABG after obtained informed consents to assess global and regional right ventricle function using conventional echocardiography and STE, all parameters obtained before and within 2 weeks after surgery. The ethics committee of the hospital approved the protocol.
Exclusion criteria were included patients refusal, presence of valvular disease, severe myocarditis, significant arrhythmia, uncompleted reperfusion therapy, previous pacemaker implantation, very poor image quality and patients on drugs that affect RV function.

All cases were subjected to detailed medical history (including name, age, sex, history of (hypertension, diabetes mellitus (DM), smoking, dyslipidemia, previous myocardial infarction(MI)), full physical examination, 12 leads ECG, routine laboratory tests including (complete blood picture, liver functions, renal functions and lipid profile) and echocardiography either conventional echocardiography or STE.

\section{Conventional echocardiography}

All 2 dimensional (2D) and tissue doppler imaging (TDI) measurements obtained using vivid 7 dimensions ultrasound frame work (GE health care, USA, WI). TAPSE was measured using $\mathrm{M}$ mode at lateral tricuspid annulus in apical 4 chamber view. Modified apical 4 chamber view focused on RV used to measure RV area by tracing the RV endocardium during systole and diastole to calculate RVFAC. RV inflow dimensions (basal and mid cavity of RV) measured in apical 4 chamber view and subcostal view as well. Pulsed wave doppler used to measure RVS' in subcostal 4 chamber view by placing TDI sample volume at lateral tricuspid annulus. RVSV measured by calculated volumes (end systolic volume (ESV) and end diastolic volume (EDV)) and heart rate (HR).

\section{Speckle tracking echocardiography}

An imaging modality that analyzes the movement of heart muscles by use of normally found speckle pattern in the heart when exposed to ultrasound beam and is considered a noninvasive tool of identification of vectors and velocity, each region of the myocardium has a unique speckle pattern that allows the region to be traced from one frame to the next. After that this can be tracked cadre to cadre and finally translated into angle-independent images. That new modality provides quantitative and qualitative information about myocardial segments deformation and motion.

Dynamic 2D ultrasound images of 3 cardiac cycles obtained at frame rates of greater than $60 \mathrm{~Hz}$ obtained and examined using altered programming with Echo PAC work station (general health care) to assess the following; Longitudinal strain of RV to analyze the free wall and interventricular septum deformation and motion (both in apical, mid cavity, basal segments) in apical 4 chamber view, RVGLS measured by averaging values measured at RV free wall and septum, also circumferential strain of RV to analyze the RV lateral free wall (anterolateral and inferolateral segments) and interventricular septum (anterior and inferior segments).

\section{Statistical analysis}

Data analysis was performed using the software SPSS (Statistical Package for the Social Sciences) version 20. 
Quantitative variables were described by their means and standard deviations. Categorical variables were described by their absolute frequencies. Kolmogorov-Smirnov (distribution-type) and Levene (homogeneity of variances) tests were used to confirm suppositions for use in parametric tests. Paired sample t test was used to compare change in one group at two points of time. The level statistical significance was set at $5 \%(p<0.05)$.

\section{Results}

In this study, male represented $61 \%$ of the studied patients while female patients were $39 \%$. Age of them ranged from 38 to 78 years with mean 57.02 years (Table 1 ).

Regarding risk factors for ischemic heart disease (IHD), $60 \%$ had hypertension, $57 \%$ were diabetic, $62 \%$ were smokers and 59\% had dyslipidemia. Only 18\% reported past history of MI (Table 2).

Twenty one percent of the studied patients had left internal mammary artery (LIMA) graft to left anterior disending (LAD) artery, $31 \%$ had both LIMA graft to LAD and saphenous vein graft(SVG) to obtuse marginal artery (OM) and $48 \%$ had LIMA graft to LAD, SVG to OM, and SVG to right coronary artery (RCA) (Table 3).

By conventional echocardiography there was statistically significant decrease in RVS' from $(12.76 \pm 1.72)$ to $(7.33 \pm$ $1.71)$ and TAPSE from $(22.8 \pm 3.99)$ to $(13.77 \pm 4.63)$ among the studied patients after CABG. While there was significant increase in RVFAC from $(44.69 \pm 3.25)$ to $(49.01 \pm 3.36)$. On the other hand, there was non-significant change in RVEDD at mid-cavity from $(26.37 \pm 2.72)$ to $(26.53 \pm 2.72)$ and basal segment from $(36.05 \pm 2.98)$ to $(36.29 \pm 3.04)$, RVSV from $(65.44 \pm 7.02)$ to $(65.85 \pm 6.86)$ or RMPI from $(0.491 \pm 0.088)$ to $(0.498 \pm 0.086)$ (Table 4$)$.

By STE There was statistically significant decrease in RVGLS from (-20.63 to -14.1) after CABG. There was statistically significant decrease in right ventricle free wall longitudinal strain [apical decreased from (-23.73 to -13.7$)$, mid-cavity decreased from (-25.76 to -11.53$)$, basal decreased from $(-20.39$ to -10.13$)$ and lateral wall declined from $(-23.01$ to -9.13)]. There was statistically significant decrease in interventricular septum longitudinal strain [apical decreased from $(-19.77$ to -10.06$)$, mid-cavity decreased from $(-17.81$ to $-10.87)$ and basal decreased from (-15.89 to -11.13$)]$. There was statistically significant increase in RV circumferential strain of lateral free wall from (-12.04 to -16.21), while there is non-significant change in RV circumferential strain of septum from $(-19.77 \pm 4.86)$ to $(-20.37 \pm 5.14)$ (Table 5).

\section{Discussion}

RV dysfunction commonly developed and reported in $>25 \%$ of patients after cardiac surgery. Either intraoperative or post-operative RV dysfunction highly associated with postoperative mortality [8].

\begin{tabular}{|c|c|c|}
\hline Demographic data & $N=100$ & $\%$ \\
\hline \multicolumn{3}{|c|}{ Gender } \\
\hline Male & 61 & 61 \\
\hline Female & 39 & 39 \\
\hline \multicolumn{3}{|c|}{ Age (years) } \\
\hline Mean \pm SD & $57.02 \pm 8.3$ & \\
\hline Range & $38-78$ & \\
\hline
\end{tabular}

Table 2: Distribution of the studied patients according to risk factors for coronary artery disease.

\begin{tabular}{|c|c|c|}
\hline Risk factors & $\boldsymbol{N}=\mathbf{1 0 0}$ & $\%$ \\
\hline Hypertension & 60 & 60 \\
\hline Diabetes mellitus & 57 & 57 \\
\hline Smoking & 62 & 62 \\
\hline Dyslipidemia & 59 & 59 \\
\hline Previous MI & 18 & 18 \\
\hline
\end{tabular}

Table 3: Distribution of the studied patients according to performed graft.

\begin{tabular}{|c|c|c|}
\hline Graft & $\boldsymbol{N}=\mathbf{1 0 0}$ & $\%$ \\
\hline LIMA to LAD & 21 & 21 \\
\hline LIMA to LAD, SVG to OM & 31 & 31 \\
\hline LIMA to LAD, SVG to OM, SVG to RCA & 48 & 48 \\
\hline
\end{tabular}

Table 4: Conventional echocardiography parameters among the studied patients before and after CABG.

\begin{tabular}{|c|c|c|c|}
\hline Parameters & Before CABG & After CABG & $P^{*}$ \\
\hline & Mean \pm SD & \multicolumn{2}{|c|}{ Mean \pm SD } \\
\hline RVEDD basal (mm) & $36.05 \pm 2.98$ & $36.29 \pm 3.04$ & 0.051 \\
\hline RVEDD midcavity $(\mathrm{mm})$ & $26.37 \pm 2.72$ & $26.53 \pm 2.72$ & 0.063 \\
\hline $\operatorname{RVS}^{\prime}(\mathrm{mm} / \mathrm{s})$ & $12.76 \pm 1.72$ & $7.33 \pm 1.71$ & $<0.001^{*}$ \\
\hline RVSV (ml) & $65.44 \pm 7.02$ & $65.58 \pm 6.86$ & 0.341 \\
\hline RVFAC (\%) & $44.69 \pm 3.25$ & $49.01 \pm 3.36$ & $<0.001^{*}$ \\
\hline TAPSE $(\mathrm{mm})$ & $22.8 \pm 3.99$ & $13.77 \pm 4.63$ & $<0.001^{*}$ \\
\hline RMPI & $0.491 \pm 0.088$ & $0.498 \pm 0.086$ & 0.583 \\
\hline
\end{tabular}

RVEDD; Right Ventricle End Diastolic Diameter; RVS'Peak: Right Ventricle Systolic Velocity; RVSV: Right Ventricle Stroke Volume; RVFAC: Right Ventricle Fractional Area Change; TAPSE: Tricuspid Annular Plane Systolic Excursion; RMPI: Right Myocardial Performance Index; CABG: Coronary Artery Bypass Graft; \#p for paired sample $t$ test. *denote significant.

Table 5: Speckle tracking echocardiography parameters among the studied patient before and after CABG.

\begin{tabular}{|c|c|c|c|}
\hline Parameters & Pre CABG & Post CABG & $P^{*}$ \\
\hline & Mean \pm SD & \multicolumn{2}{c|}{ Mean \pm SD } \\
\hline RVGLS (\%) & $-20.63 \pm 2.53$ & $-14.1 \pm 3.25$ & $<0.001^{*}$ \\
\hline \multicolumn{4}{|c|}{ RV free wall longitudinal strain (\%) } \\
\hline Apical & $-23.73 \pm 2.21$ & $-13.7 \pm 1.96$ & $<0.001^{*}$ \\
\hline Mid & $-25.76 \pm 3.08$ & $-11.53 \pm 3.41$ & $<0.001^{*}$ \\
\hline Basal & $-20.39 \pm 4.41$ & $-10.13 \pm 2.74$ & $<0.001^{*}$ \\
\hline Lateral wall & $-23.01 \pm 3.74$ & $-9.13 \pm 1.99$ & $<0.001^{*}$ \\
\hline & Interventricular septum longitudinal strain $(\%)$ & \\
\hline Apical & $-19.77 \pm 5.38$ & $-10.06 \pm 4.54$ & $<0.001^{*}$ \\
\hline Mid & $-17.81 \pm 3.69$ & $-10.87 \pm 3.94$ & $<0.001^{*}$ \\
\hline Basal & $-15.89 \pm 3.14$ & $-11.13 \pm 3.9$ & $<0.001^{*}$ \\
\hline & RV circumferential strain (\%) & \\
\hline Lateral free wall & $-12.04 \pm 1.91$ & $-16.21 \pm 2.75$ & $<0.001^{*}$ \\
\hline Septum & $-19.77 \pm 4.86$ & $-20.37 \pm 5.14$ & 0.213 \\
\hline
\end{tabular}

RVGLS: Right Ventricle Global Longitudinal Strain; CABG: Coronary Artery Bypass Graft; $" p$ for paired sample t test. *denote significant 
In this study, male represented $61 \%$ of the studied patients while female patients are $39 \%$, age of them ranged from 38 to 78 years with mean 57.02 years.

Also we are in line with El-Adawy, et al. [9], who studied fifty consecutive patients with IHD of them (34 (68\%) male and $16(32 \%)$ female). The mean age of the study population was $52.92 \pm 9.97$ years [9].

A total of 4,024 patients performed CABG included in the study by Varma, et al. [10], their mean age was $57 \pm 9.6$ years. The incidence of female patients undergoing surgery was the minority of cases (12.6\%) [10].

Also in harmony with us among fifty patients were included in the Korshin, et al, [11], study, the gender distribution revealed more male than female patients [11].

Although among twenty-four patients undergoing elective CABG were enrolled by Bitcon and Tousignant, There were 18 male and three female patients which similar to our results. Mean (SD) age was higher than our study as the mean value was 70 year [12].

In this study, $21 \%$ of the studied patients had LIMA graft to LAD artery, 31\% had both LIMA graft to LAD artery and SVG to OM artery and $48 \%$ had LIMA graft to LAD artery, SVG to OM artery, and SVG to RCA artery.

Similarly, in study by Gozdzik, et al. [14], 69 patients undergoing elective CABG were included. 55 of them had three-vessel disease. The most of patients received three grafts and in all LIMA graft to the LAD artery was used [14].

El-Adawy, et al. [9], reported that, the most common diseased coronary vessel was LAD in $(49 \%)$ of patients followed by (34\%) had RCA \& (17\%) had OM [9].

In this study, regarding the risk factors for IHD, $60 \%$ had hypertension, $57 \%$ were diabetics, $62 \%$ were smokers and $59 \%$ had dyslipidemia. Only 18\% reported past history of MI.

This was in agreement with Varma, et al. [10], study in which the patients at the time of CABG surgery showed that hypertension was the commonest risk factors [10].

Also, we are in line with El-Adawy, et al. [9], who studied fifty patients with IHD. $32 \%$ of them were diabetic, $40 \%$ were hypertensive, $10 \%$ were smoker and $30 \%$ of patients were complaining of dyslipidemia [9].

In this study, by conventional echocardiography there was statistically significant decrease in RVS' from $(12.76 \pm 1.72)$ to $(7.33 \pm 1.71)$ and TAPSE from $(22.8 \pm 3.99)$ to $(13.77 \pm$ 4.63) among the studied patients after CABG. While there was significant increase in RVFAC from (44.69 \pm 3.25$)$ to (49.01 \pm $3.36)$. On the other hand, there was non-significant change in right ventricle end diastolic diameter (RVEDD) measured at mid-cavity and basal segment, RVSV or RMPI after CABG.
The possible reason of deformation of RV geometry, reduction of TAPSE and RVS' after open heart surgery was still unclear. Changed contraction pattern of the interventricular septum, pericardiotomy mechanical effects, or postoperative adhesions of the right ventricle may be causative factors. Some theories were proposed to explain this dysfunction as pericardial opening, injury to the right atrium during cannulation, incomplete myocardial protection, and adhesions between the RV and nearby mediastinal structures $[15,16]$, right ventricular ejection fraction (RVEF) is unchanged and cardiac output (COP) is not reduced [17].

In agreement with our study, 50 patients underwent CABG were included in a study by Korshin, et al. [11], who found significant reduction in TAPSE after surgery [11].

A second study done by Unsworth, et al. [18], who examined a group of 34 patients undergoing conventional on pump \& robotic CABG, They found significant reduction in TAPSE after CABG. But in contrast to us, they found that a significant reduction in RVSV only in patients undergoing conventional on-pump CABG [18].

In concordance with our results, Hashemi, et al. [19], found that, TAPSE decreaesd significantly when matching the values from before to after CABG $(23.9 \pm 4.46$ vs $14.6 \pm 3.67$, $p<0.001)$. In contrast to us, Hashemi, et al. [19], found that, RVS' also decreased after CABG $(11.9 \pm 2.40$ vs $8.5 \pm 1.93, p<$ 0.001) [19].

Another study by Rösner, et al. [17], indicated that postCABG tricuspid TAPSE was markedly reduced, but there was unchanged RVFAC [17].

Morever, Joshi, et al. [20], noted that after CABG, although there was a reduction in TAPSE, there was reduction of RVSV assessed by echocardiography with an improvement in RV function assessed by RMPI [20].

Also, after off-pump CABG, Khani, et al. [21], reported that the reduction in TAPSE not belonged to the cardiopulmonary bypass itself [21].

Our results were in line with Unsworth, et al. [22] who conducted echocardiography in 33 patients before and after elective CABG , TAPSE estimates decline significantly after the surgery by $58 \%$, $(p<0.0001)$ [22].

Also, among forty six patients who were accepted for CABG were included in a study by Hashemi, et al. [19], on the impact of CABG on RV function, TAPSE reduced substantially after CABG $(p<0.001)$, On contrary, RMPI improved significantly $(p<0.001)$ with significant decline in RVS' was also observed following CABG $(p<0.001)$ [19].

In disagreement with our study, among twenty-four patients undergoing elective CABG were enrolled by Bitcon, et al. [12], there was significant change in post chest closure 
values of RVFAC comparing to pre-pericardiotomy. On the other hand, there was a significant reduction in postoperative value of TAPSE \& RVSV [12].

In contrast to our study, Larrazet, et al. [23], found that RVFAC remained unchanged despite there was a decline in RVSV after CABG [23].

In this study, by STE there was statistically significant decrease in RVGLS from (-20.63 to -14.1) after CABG. There is statistically significant decrease in RV free wall longitudinal strain [apical decreased from (-23.73 to -13.7$)$, mid-cavity decreased from (-25.76 to -11.53$)$, basal decreased from $(-20.39$ to -10.13$)$ and lateral wall declined from $(-23.01$ to -9.13)]. There is statistically significant decrease in interventricular septum longitudinal strain [apical decreased from (-19.77 to -10.06$)$, mid-cavity decreased from $(-17.81$ to -10.87) and basal decreased from ( -15.89 to -11.13$)]$. There was statistically significant increase in RV circumferential strain of lateral free wall from $(-12.04$ to -16.21$)$ while there was non-significant change in RV circumferential strain of septum from $(-19.77 \pm 4.86)$ to $(-20.37 \pm 5.14)$.

Our results were in agreement with previous study after cardiac surgery, there was decline in the longitudinal contraction of the RV lateral free wall [17].

Similarly, Gozdzik, et al. [14], enrolled 69 patients scheduled for CABG, they observed a significant decrease in the RVGLS post operatively [14].

In line with our results, as shown by Rong, et al. [24], RV free wall global longitudinal strain decreased significantly post operatively ( $p<0.05$ for all). With no significant change in post-operative interventricular septal deformation ( $p=$ $0.23)[24]$.

Similar finding was observed by Rong, et al. [24], who showed a significant reduction in RVGLS $(p<0.001)$ [24].

Similar to our results, among twenty-four patients undergoing elective CABG were enrolled by Bitcon, et al. [12], there was a significant decrease in in RV free wall strain [12].

While some studies have reported deterioration in global $\mathrm{RV}$ function and reduced longitudinal RV movement [25], other studies using other modalities for assessment of RV function had reported preserved global RV function despite a post-surgical reduction in RV longitudinal function [26].

Maffessanti, et al. [16], and Lindqvist, et al. [27], studies observed that no significant changes in the interventricular septum postoperative. The mechanism of these controversial findings is vague and may be due to a paradoxical movement of the septum after CABG, as well as cardiac injury due to ischemia [16,27].

\section{Conclusion}

In conclusion, this study revealed high prevalence of most of the cardiovascular risk factors especially diabetes, hypertension and dyslipidemia in patients underwent CABG. By conventional echocardiography, TAPSE and RVS' were significantly decreased while RVFAC was significantly increased than its initial value in all patients after CABG. By STE, Global, free wall and interventricular septum longitudinal strain of RV were decreased significantly while circumferential strain of the RV lateral wall increased significantly. Distorted right ventricle geometry after CABG can lead to altered deformation parameters, in other words longitudinal functional parameters may underestimate $\mathrm{RV}$ function and the decrease in RVGLS was compensated by increase in circumferential strain of lateral free wall of RV without change in RVSV or RMPI. Therefor changes in deformation parameters should always be interpreted in relation to change in geometry.

\section{Limitations}

The influence of RV dysfunction on post-operative morbidity and mortality is not estimated. This study not evaluated intraoperative RV function rather than only after 2 weeks postoperatively. Also, as a cross sectional study we couldn't determine effects of risk factors for coronary artery disease on outcome following CABG. The lack of other independent diagnostic modalities as magnetic resonance imaging, Radial strain of RV not evaluated. The effect of the treatment on the prevalence of risk factors and on the RV function are not studied. Additionally, limited follow- up period beside the low sample size were the most important study limitations.

\section{References}

1. Lozano R, Naghavi M, Foreman K, Lim S, Shibuya K, et al. Global and regional mortality from 235 causes of death for 20 age groups in 1990 and 2010: a systematic analysis for the Global Burden of Disease Study 2010. Lancet. 2012; 380: 2095-2128.

PubMed: https://www.ncbi.nlm.nih.gov/pubmed/23245604

2. Habib RH, Dimitrova KR, Badour SA, Yammine MB, El-Hage-Sleiman $A K M$, et al. CABG versus PCl: greater benefit in long-term outcomes with multiple arterial bypass grafting. J Am Coll Cardiol. 2015; 66: 1417-1427.

PubMed: https://www.ncbi.nlm.nih.gov/pubmed/26403338

3. Ivanov J, Borger MA, Tu JV, Rao V, David TE. Mid-term outcomes of off-pump versus on-pump coronary artery bypass graft surgery. Can $\mathrm{J}$ Cardiol. 2008; 24: 279-284.

PubMed: https://www.ncbi.nlm.nih.gov/pubmed/18401468

4. Jones EL, Weintraub WS. The importance of completeness of revascularization during long-term follow-up after coronary artery operations. J Thorac Cardiovasc Surg. 1996; 112: 227-237. PubMed: https://www.ncbi.nlm.nih.gov/pubmed/8751484

5. Dalen $\mathrm{H}$, Thorstensen $\mathrm{A}$, Aase $\mathrm{SA}$, Ingul CB, Torp $\mathrm{H}$, et al. Segmental and global longitudinal strain and strain rate based on echocardiography of 1266 healthy individuals: the HUNT study in Norway. Eur J Echocardiogr. 2010; 11: 176-183.

PubMed: https://www.ncbi.nlm.nih.gov/pubmed/19946115

6. Stoylen A, Heimdal A, Bjornstad K, Wiseth R, Vik-Mo H, et al. Strain 
rate imaging by ultrasonography in the diagnosis of coronary artery disease. J Am Soc Echocardiogr. 2000; 13: 1053-1064.

PubMed: https://www.ncbi.nlm.nih.gov/pubmed/11119272

7. Lucreziotti S, Gavazzi A, Scelsi L, Inserra C, Klersy C, et al. Five-minute recording of heart rate variability in severe chronic heart failure: correlates with right ventricular function and prognostic implications. Am Heart J. 2000; 139: 1088-1095.

PubMed: https://www.ncbi.nlm.nih.gov/pubmed/10827392

8. Bootsma IT, de Lange F, Koopmans M, Haenen J, Boonstra PW, et al. Right ventricular function after cardiac surgery is a strong independent predictor for long-term mortality. J Cardiothorac Vasc Anesth. 2017; 31: 1656-1662.

PubMed: https://www.ncbi.nlm.nih.gov/pubmed/28416392

9. El-Adawy A, Salem M, Mahfouz E, El-Safty E, Abdelsamad A, et al. Assessment of Right Ventricular Functions in Patients with Ischemic Heart Disease Before and After Percutaneous Coronary Intervention using Colored Tissue Doppler Imaging. 2018; 9: 196-201.

10. Varma PK, Kundan S, Ananthanarayanan C, Panicker VT, Pillai VV, et al. Demographic profile, clinical characteristics and outcomes of patients undergoing coronary artery bypass grafting-retrospective analysis of 4,024 patients. Indian Journal of Thoracic and Cardiovascular Surgery. 2014; 30: 272-277.

11. Korshin A, Grønlykke L, Nilsson JC, Møller-Sørensen H, Ihlemann N, et al. Tricuspid annular plane systolic excursion is significantly reduced during uncomplicated coronary artery bypass surgery: A prospective observational study. J Thorac Cardiovasc Surg. 2019; 158: 480-489. PubMed: https://www.ncbi.nlm.nih.gov/pubmed/30527715

12. Bitcon CJ, Tousignant C. The effect of pericardial incision on right ventricular systolic function: a prospective observational study. Can J Anesth. 2017; 64: 1194-1201.

PubMed: https://www.ncbi.nlm.nih.gov/pubmed/28983853

13. Cornwell LD, Omer S, Rosengart T, Holman WL, Bakaeen FG. Changes over time in risk profiles of patients who undergo coronary artery bypass graft surgery: the Veterans Affairs Surgical Quality Improvement Program (VASQIP). JAMA Surg. 2015; 150: 308-315. PubMed: https://www.ncbi.nlm.nih.gov/pubmed/25671647

14. Gozdzik A, Letachowicz K, Grajek BB, Plonek T, Obremska M, et al. Application of strain and other echocardiographic parameters in the evaluation of early and long-term clinical outcomes after cardiac surgery revascularization. BMC Cardiovasc Disord. 2019; 19: 189. PubMed: https://www.ncbi.nlm.nih.gov/pubmed/31382900

15. Hedman A, Alam M, Zuber E, Nordlander R, Samad BA. Decreased right ventricular function after coronary artery bypass grafting and its relation to exercise capacity: A tricuspid annular motion-based study. J Am Soc Echocardiogr. 2004; 17: 126-131.

PubMed: https://www.ncbi.nlm.nih.gov/pubmed/14752486

16. Lindqvist $P$, Holmgren A, Zhao Y, Henein MY. Effect of pericardial repair after aortic valve replacement on septal and right ventricular function. Int J Cardiol. 2012; 155: 388-393.

PubMed: https://www.ncbi.nlm.nih.gov/pubmed/21112100

17. Rösner A, Avenarius $D$, Malm S, Iqbal A, Schirmer $H$, et al. Changes in right ventricular shape and deformation following coronary artery bypass surgery-insights from echocardiography with strain rate and magnetic resonance imaging. Echocardiography. 2015; 32: 1809-1820. PubMed: https://www.ncbi.nlm.nih.gov/pubmed/26010320

18. Unsworth B, Casula RP, Yadav H, Baruah R, Hughes AD, et al. Contrasting effect of different cardiothoracic operations on echocardiographic right ventricular long axis velocities, and implications for interpretation of post-operative values. Int J Cardiol. 2013; 165: 151-160. PubMed: https://www.ncbi.nlm.nih.gov/pubmed/21917325

19. Hashemi N, Brodin LA, Hedman A, Samad B, Alam M. Improved right ventricular index of myocardial performance in the assessment of right ventricular function after coronary artery bypass grafting. Interact Cardiovasc Thorac Surg. 2018; 26: 798-804.

PubMed: https://www.ncbi.nlm.nih.gov/pubmed/29304238

20. Joshi SB, Roswell RO, Salah AK, Zeman PR, Corso PJ, et al. Right ventricular function after coronary artery bypass graft surgery-a magnetic resonance imaging study. Cardiovasc Revasc Med. 2010; 11: 98-100.

PubMed: https://www.ncbi.nlm.nih.gov/pubmed/20347799

21. Khani $M$, Hosseintash $M$, Foroughi $M$, Naderian $M$, Khaheshi $I$. Assessment of the effect of off-pump coronary artery bypass (OPCAB) surgery on right ventricle function using strain and strain rate imaging. Cardiovasc Diagn Ther. 2016; 6: 138-143.

PubMed: https://www.ncbi.nlm.nih.gov/pubmed/27054103

22. Unsworth B, Casula RP, Kyriacou AA, Yadav H, Chukwuemeka A, et al. The right ventricular annular velocity reduction caused by coronary artery bypass graft surgery occurs at the moment of pericardial incision. Am Heart J. 2010; 159: 314-322.

PubMed: https://www.ncbi.nlm.nih.gov/pubmed/20152232

23. Larrazet F, Czitrom D, Laborde F, Bouabdallah K, Folliguet T. Decreased right ventricular lateral wall velocities early after cardiac surgery. Echocardiography. 2011; 28: 438-441.

PubMed: https://www.ncbi.nlm.nih.gov/pubmed/21504466

24. Rong LQ, Yum B, Abouzeid C, Palumbo MC, Brouwer LR, et al. Echocardiographic predictors of intraoperative right ventricular dysfunction: a 2D and speckle tracking echocardiography study. Cardiovasc Ultrasound. 2019; 17: 11.

PubMed: https://www.ncbi.nlm.nih.gov/pubmed/31174537

25. Kempny A, Diller GP, Kaleschke G, Orwat S, Funke A, et al. Impact of transcatheter aortic valve implantation or surgical aortic valve replacement on right ventricular function. Heart. 2012; 98: 1299-1304. PubMed: https://www.ncbi.nlm.nih.gov/pubmed/22689711

26. Tamborini G, Muratori M, Brusoni D, Celeste F, Maffessanti F, et al. Is right ventricular systolic function reduced after cardiac surgery? A twoand three-dimensional echocardiographic study. Eur J Echocardiogr. 2009; 10: 630-634.

PubMed: https://www.ncbi.nlm.nih.gov/pubmed/19252190

27. Maffessanti F, Gripari P, Tamborini G, Muratori M, Fusini L, et al Evaluation of right ventricular systolic function after mitral valve repair: a two-dimensional Doppler, speckle-tracking, and three-dimensional echocardiographic study. J Am Soc Echocardiogr. 2012; 25: 701-708. PubMed: https://www.ncbi.nlm.nih.gov/pubmed/22542273 